\title{
Light flavon signals at electron-photon colliders
}

\author{
Yu Muramatsu, ${ }^{1, *}$ Takaaki Nomura, ${ }^{2, \dagger}$ Yusuke Shimizu, ${ }^{3, *}$ and Hiroshi Yokoya ${ }^{4, \S}$ \\ ${ }^{1}$ Institute of Particle Physics and Key Laboratory of Quark and Lepton Physics (MOE), \\ Central China Normal University, Wuhan, Hubei 430079, People's Republic of China \\ ${ }^{2}$ School of Physics, KIAS, Seoul 130-722, Republic of Korea \\ ${ }^{3}$ Graduate School of Science, Hiroshima University, Higashi-Hiroshima 739-8526, Japan \\ ${ }^{4}$ Quantum Universe Center, KIAS, Seoul 130-722, Republic of Korea
}

(Received 17 October 2017; published 8 January 2018)

\begin{abstract}
Flavor symmetries are useful to realize fermion flavor structures in the standard model (SM). In particular, the discrete $A_{4}$ symmetry is used to realize lepton flavor structures, and some scalars — called flavons-are introduced to break this symmetry. In many models, flavons are assumed to be much heavier than the electroweak scale. However, our previous work showed that a flavon mass around $100 \mathrm{GeV}$ is allowed by experimental constraints in the $A_{4}$ symmetric model with a residual $Z_{3}$ symmetry. In this paper, we discuss collider searches for such a light flavon $\varphi_{T}$. We find that electron-photon collisions at the International Linear Collider have advantages for searching for these signals. In electron-photon collisions, flavons are produced as $e^{-} \gamma \rightarrow l^{-} \varphi_{T}$ and decay into two charged leptons. Then, we analyze signals of the flavor-conserving final state $\tau^{+} \tau^{-} e^{-}$and the flavor-violating final states $\tau^{+} \mu^{-} \mu^{-}$and $\mu^{+} \tau^{-} \tau^{-}$by carrying out numerical simulations. For the former final state, SM background can be strongly suppressed by imposing cuts on the invariant masses of final-state leptons. For the latter final states, SM background is extremely small, because in the SM there are no such flavor-violating final states. We then find that sufficient discovery significance can be obtained, even if flavons are heavier than the lower limits from flavor physics.
\end{abstract}

DOI: 10.1103/PhysRevD.97.015003

\section{INTRODUCTION}

The standard model (SM) particles were completed with the discovery of the Higgs boson. However, the origin of the generation and flavor structure of the SM fermions is not clear. In order to explain the flavor structure of the SM fermions, we can introduce flavor symmetries and scalar fields-so-called "flavons." After flavons take vacuum expectation values (VEVs), flavor symmetries are broken and SM fermions obtain a flavor structure.

In the SM, neutrinos are massless. However, neutrino oscillation experiments reveal neutrino mass-squared differences and large lepton mixing angles [1-5]. In order to explain large lepton mixing angles, many authors have studied the lepton flavor structure by using non-Abelian discrete symmetries as a flavor symmetry (for a review, see Refs. [6-9]). The non-Abelian discrete flavor symmetry can

\footnotetext{
yumura@mail.ccnu.edu.cn

nomura@kias.re.kr

*yu-shimizu@hiroshima-u.ac.jp

§hyokoya@kias.re.kr
}

Published by the American Physical Society under the terms of the Creative Commons Attribution 4.0 International license. Further distribution of this work must maintain attribution to the author(s) and the published article's title, journal citation, and DOI. Funded by SCOAP ${ }^{3}$. easily derive the large lepton mixing angles, e.g., tribimaximal mixing (TBM) which is a simple paradigm of the lepton mixing matrix. Indeed, Altarelli and Feruglio (AF) proposed an $A_{4}$ flavor model $[10,11]$ which introduces gauge singlet flavons in addition to the $S U(2)$ doublet SM Higgs field. In the AF model, the lepton mixing matrix is the exact TBM one. However, the observation of the nonzero reactor angle forces us to study the deviation from the TBM or study other flavor paradigms. In Ref. [12], the authors predicted the nonzero reactor angle with broken TBM by adding an extra flavon to the AF model.

From the experimental point of view, flavor symmetries have not been confirmed yet. Many authors have tried to predict the Dirac $C P$-violating phase, Majorana phases, and effective mass for the neutrinoless double beta decay, which could be indirect evidence for flavons. In Refs. [13-16], the authors discussed the mass restriction on the flavons which mix with the SM Higgs, from lepton flavor violation (LFV) and collider physics. On the other hand, in our previous work [17] we studied experimental constraints for flavons which do not mix with the SM Higgs. From LFV constraints the lower limit of the flavon masses is around $60 \mathrm{GeV}$. Such a light flavon mass limit comes from the residual $Z_{3}$ symmetry.

Because of the light flavon mass limit we can expect direct flavon signals at colliders. First, we examine this 
TABLE I. The production cross sections at the LHC for each flavon, where $\sqrt{s}=14 \mathrm{TeV}$ and the flavon masses are $v_{T}=2 m_{\varphi_{T 1}}=m_{\varphi_{T 2}}=m_{\varphi_{T 3}}=65 \mathrm{GeV}$. In this calculation we use MADGRAPH with default momentum and rapidity cuts.

\begin{tabular}{|c|c|c|c|c|c|c|}
\hline $\begin{array}{l}\text { Final state } \\
\text { Cross section [fb] }\end{array}$ & $\begin{array}{c}\varphi_{T 1} \tau^{-} \bar{\nu}_{\tau} \\
2.2\end{array}$ & $\begin{array}{r}\varphi_{T 1} \tau^{-} \tau^{+} \\
1.5 \times 10^{-1}\end{array}$ & $\begin{array}{r}\varphi_{T 2} \tau^{-} \bar{\nu}_{\mu} \\
1.7 \times 10^{-5}\end{array}$ & $\begin{array}{r}\varphi_{T 2} \tau^{-} \mu^{+} \\
8.4 \times 10^{-6}\end{array}$ & $\begin{array}{r}\varphi_{T 3} \tau^{-} \bar{\nu}_{e} \\
1.7 \times 10^{-5}\end{array}$ & $\begin{array}{r}\varphi_{T 3} \tau^{-} e^{+} \\
8.4 \times 10^{-6}\end{array}$ \\
\hline
\end{tabular}

possibility at the Large Hadron Collider (LHC). However, as we will show it is hard to find flavon signals at the LHC. Then, we examine other possibilities at lepton colliders. In particular, we find that an electron-photon collider has many advantages for searching for flavon signals. The photon beam is obtained from backscattered Compton photons $[18,19]$, and therefore electron-photon collisions could be realized at future lepton colliders. The possibility of an electron-photon collider has been discussed for a long time [18,20,21]. The phenomenology of electron-photon collisions has been discussed in the context of the International Linear Collider (ILC) [22,23]. In this paper, we show that sufficient discovery significance can be obtained in electron-photon collisions at the ILC.

This paper is organized as follows. In Sec. II, we summarize the modified AF model. In Sec. III, we examine flavon signals at the LHC. In Sec. IV, we show the advantages for searching for flavon signals in electronphoton collisions at the ILC. Moreover, we show flavon signals in flavor-conserving processes and flavor-violating processes. Our summary and discussion are given in Sec. V.

\section{II. $A_{4}$ FLAVOR MODEL}

In this section, we briefly summarize the modified AF model [17]. First of all, we discuss the mass of the flavon $\phi_{T}$ which is a triplet under the $A_{4}$ group and couples to the charged leptons. One of the solutions of the potential minimum in Refs. $[17,24]$ leads to the following value for the VEV of $\phi_{T}, v_{T}$ :

$$
\left\langle\phi_{T}\right\rangle=v_{T}(1,0,0), \quad v_{T}=\frac{3 M}{2 g},
$$

where $M$ is a mass parameter and $g$ is a trilinear coupling in the flavon potential. By using the VEV in Eq. (1), we can calculate the mass of the flavon $\phi_{T}$. We expand the flavon field around the VEV $v_{T}$ as

$$
\phi_{T}=\left(\phi_{T 1}, \phi_{T 2}, \phi_{T 3}\right) \rightarrow\left(v_{T}+\varphi_{T 1}, \varphi_{T 2}, \varphi_{T 3}\right)
$$

where $\varphi_{T i}$ are complex scalar fields. Then, the masses of the scalar fields $m_{\varphi_{T i}}$ are obtained as

$$
\left(m_{\varphi_{T 1}}^{2}, m_{\varphi_{T 2}}^{2}, m_{\varphi_{T 3}}^{2}\right)=\left(2 M^{2}, 8 M^{2}, 8 M^{2}\right),
$$

and $\varphi_{T i}$ do not mix with each other. Hereafter, we assume that $v_{T}=2 m_{\varphi_{T 1}}=m_{\varphi_{T 2}}=m_{\varphi_{T 3}}$ for simplicity.

Next, we discuss the charged lepton sector. The Lagrangian, including the SM Yukawa and flavon Yukawa interactions, is written as follows [17]:

$$
\begin{aligned}
\mathcal{L}_{\ell}= & y_{e}\left(\phi_{T} \bar{l}\right) e_{R} h_{d} / \Lambda+y_{\mu}\left(\phi_{T} \bar{l}\right) \mu_{R} h_{d} / \Lambda \\
& +y_{\tau}\left(\phi_{T} \bar{l}\right) \tau_{R} h_{d} / \Lambda+\text { H.c. },
\end{aligned}
$$

where $y_{\alpha}(\alpha=e, \mu, \tau)$ are Yukawa couplings, $\Lambda$ is an $A_{4}$ cutoff scale, and $h_{d}$ is an $S U(2)$ doublet Higgs. The lefthanded lepton doublets $l=\left(l_{e}, l_{\mu}, l_{\tau}\right)$ are assigned to triplets under the $A_{4}$ group, while the right-handed charged leptons $e_{R}, \mu_{R}$, and $\tau_{R}$ are assigned to singlets denoted as $\mathbf{1}$, $\mathbf{1}^{\prime \prime}$, and $\mathbf{1}^{\prime}$, respectively. After expanding $\phi_{T}$ around $v_{T}$ as in Eq. (2) and taking the VEV of the $S U(2)$ doublet Higgs $h_{d}$ (denoted as $v_{d}$ ), the charged lepton mass term $\mathcal{L}_{\ell}^{\text {mass }}$ is written as

$$
\begin{aligned}
& \mathcal{L}_{\ell}^{\text {mass }}=\left(\begin{array}{lll}
\bar{e}_{L} & \bar{\mu}_{L} & \bar{\tau}_{L}
\end{array}\right)\left(\begin{array}{ccc}
\frac{y_{e} v_{d}}{\Lambda} v_{T} & 0 & 0 \\
0 & \frac{y_{\mu} v_{d}}{\Lambda} v_{T} & 0 \\
0 & 0 & \frac{y_{\tau} v_{d}}{\Lambda} v_{T}
\end{array}\right)\left(\begin{array}{l}
e_{R} \\
\mu_{R} \\
\tau_{R}
\end{array}\right) \\
&+ \text { H.c. } \\
& \equiv\left(\begin{array}{lll}
\bar{e}_{L} & \bar{\mu}_{L} & \bar{\tau}_{L}
\end{array}\right)\left(\begin{array}{ccc}
m_{e} & 0 & 0 \\
0 & m_{\mu} & 0 \\
0 & 0 & m_{\tau}
\end{array}\right)\left(\begin{array}{l}
e_{R} \\
\mu_{R} \\
\tau_{R}
\end{array}\right)+\text { H.c. }
\end{aligned}
$$

In our model, charged leptons in the interaction basis are equal to those in the mass basis. Therefore, there is no mixing in the charged lepton sector at the leading-order level. Moreover, the charged lepton and flavon interaction term $\mathcal{L}_{\ell}^{\mathrm{FY}}$ is obtained as

$$
\begin{aligned}
\mathcal{L}_{\ell}^{\mathrm{FY}}= & \left(\begin{array}{lll}
\bar{e}_{L} & \bar{\mu}_{L} & \bar{\tau}_{L}
\end{array}\right)\left(\begin{array}{ccc}
\frac{m_{e}}{v_{T}} & 0 & 0 \\
0 & \frac{m_{\mu}}{v_{T}} & 0 \\
0 & 0 & \frac{m_{\tau}}{v_{T}}
\end{array}\right)\left(\begin{array}{c}
e_{R} \\
\mu_{R} \\
\tau_{R}
\end{array}\right) \varphi_{T 1} \\
& +\left(\begin{array}{lll}
\bar{e}_{L} & \bar{\mu}_{L} & \bar{\tau}_{L}
\end{array}\right)\left(\begin{array}{ccc}
0 & \frac{m_{\mu}}{v_{T}} & 0 \\
0 & 0 & \frac{m_{\tau}}{v_{T}} \\
\frac{m_{e}}{v_{T}} & 0 & 0
\end{array}\right)\left(\begin{array}{c}
e_{R} \\
\mu_{R} \\
\tau_{R}
\end{array}\right) \varphi_{T 2} \\
& +\left(\begin{array}{llll}
\bar{e}_{L} & \bar{\mu}_{L} & \bar{\tau}_{L}
\end{array}\right)\left(\begin{array}{ccc}
0 & 0 & \frac{m_{\tau}}{v_{T}} \\
\frac{m_{e}}{v_{T}} & 0 & 0 \\
0 & \frac{m_{\mu}}{v_{T}} & 0
\end{array}\right)\left(\begin{array}{c}
e_{R} \\
\mu_{R} \\
\tau_{R}
\end{array}\right) \varphi_{T 3} \\
& + \text { H.c. }
\end{aligned}
$$

We find that $\varphi_{T 1}$ exchange does not induce flavor violation, while the other flavon exchanges induce flavor violation. We also find that the couplings are fixed by charged lepton masses except for $v_{T}$. 

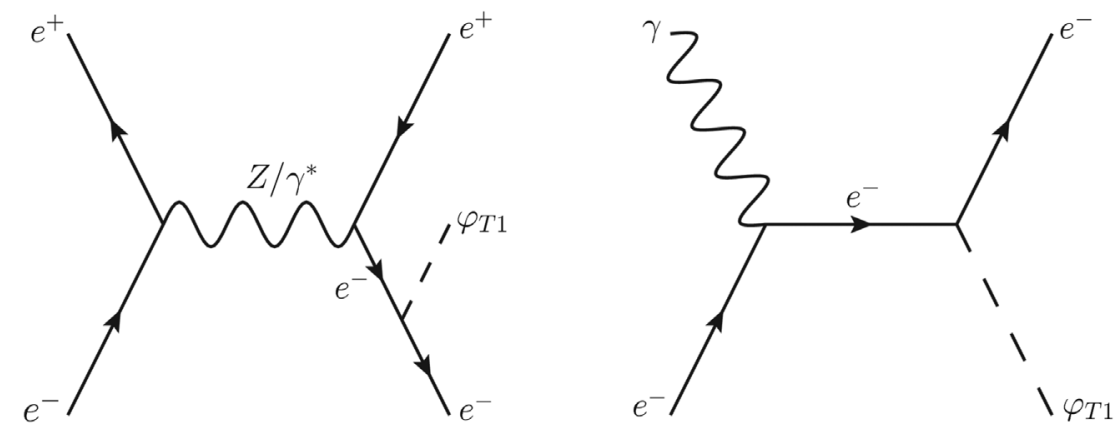

FIG. 1. One of the diagrams which produces the flavon $\varphi_{T 1}$ at an electron-positron collider (left) and an electron-photon collider (right).

Before closing this section, we mention the lower bound of the flavon mass, which was discussed in Ref. [17]. After taking the VEV of the flavon, the $A_{4}$ symmetry is broken down to the residual $Z_{3}$ symmetry. Thanks to the residual $Z_{3}$ symmetry, many lepton-flavor-violating decay modes, such as $\mu \rightarrow e \gamma$ and $\mu \rightarrow 3 e$, are forbidden. ${ }^{1}$ Because lepton-flavor-violating decay modes (which have severe constraints) are forbidden, the mass lower bound of the flavon is found to be $60 \mathrm{GeV}$ which comes from the $\tau$ three-body decay mode $\tau^{ \pm} \rightarrow \mu^{ \pm} \mu^{ \pm} e^{\mp}$. In addition, if we assume the Yukawa coupling $y_{\tau}$ to be $\mathcal{O}(1)$ the cutoff scale is typically $\mathcal{O}(10) \mathrm{TeV}$.

\section{FLAVON SIGNALS AT PROTON-PROTON COLLIDERS}

In our previous paper [17], we showed that in our model the flavon can be light, and therefore we expect flavon signals at colliders. Hence, we show the flavon production cross section at the LHC. In Table I, we summarize the flavon production cross section calculated in Ref. [17]. In this calculation we set $\sqrt{s}=14 \mathrm{TeV}$ and the flavon masses around the lower bound $v_{T}=2 m_{\varphi_{T 1}}=m_{\varphi_{T 2}}=m_{\varphi_{T 3}}=65 \mathrm{GeV}$. To calculate these values we use MADGRAPH5 [26] with default momentum and rapidity cuts. ${ }^{2}$ We find that the $\varphi_{T 1}$ production cross sections are larger than other flavon production cross sections because $\varphi_{T 1}$ is lighter than the other flavons. Moreover, $\varphi_{T 1}$ production cross sections are $O(1) \mathrm{fb}$, and therefore we can expect the $A_{4}$ flavon signals at the LHC.

Because $\varphi_{T 1}$ mainly decays into tau and antitau leptons, the expected flavon signal processes at proton-proton colliders include at least three tau leptons. In collider experiments the identification of tau leptons is more difficult than that of the other charged leptons. Therefore, we have to consider whether all tau leptons in flavon signal processes can be identified. To do so, we perform detector simulations using MadGraph5 [26], Pythia [27], and Delphes [28]. We generate events for $p p \rightarrow \tau^{-} \tau^{-} \tau^{+} \bar{\nu}_{\tau}$ and $\tau^{-} \tau^{-} \tau^{+} \tau^{+}$processes

\footnotetext{
${ }^{1}$ In some models with the $A_{4}$ symmetry, LFVs that come from $Z_{3}$-breaking effects were discussed [25].

${ }^{2}$ Because of the momentum and rapidity cuts, the values of the production cross sections are not the same as the values in Table 2 in the previous paper [17].
}

through $\varphi_{T 1}$ production and perform detector simulations based on the LHC detector performance. Then, we count the number of events in which all tau leptons are identified as tautagged jets, and find that the expected number of events is less than one for both final states when $3000 \mathrm{fb}^{-1}$ data are collected. Therefore, it is impossible to get sufficient discovery significance for our flavon at the LHC. This feature is due to the low momentum cut for tau jets which come from the flavon decay. ${ }^{3}$ In the low-flavon-mass region, although the flavon production cross section is large, charged leptons from the light flavon decay tend to have low momenta.

\section{ELECTRON-PHOTON COLLIDER}

At lepton colliders, flavons are produced by $e^{+} e^{-} \rightarrow$ $l^{+} l^{-} \varphi_{T}$ processes. As with flavon production processes at the LHC, we find that it is hard to obtain sufficient discovery significance from these flavon processes at the ILC. ${ }^{4}$ As an alternative possibility for obtaining flavon signals at lepton colliders, we consider electron-photon collisions as an option for future lepton collider experiments such as the ILC. At lepton colliders, a high-energy photon beam can be produced by Compton backscattering of laser photons with electrons. In electron-photon collisions, the lowest-order process for flavon production is $e^{-} \gamma \rightarrow \ell^{-} \varphi_{T}$. In Fig. 1, we show one of the diagrams for $\varphi_{T 1}$ production at an electron-positron collider (left) and at an electron-photon collider (right). Because of the order of QED interactions and the final-state phase-space volume, we expect the flavon production cross section to be larger at the electron-photon collider than that at the electron-positron collider. By including the decay of $\varphi_{T} \rightarrow l^{+} l^{\prime-}$ the process contains three leptons, all of which are expected to have larger momenta.

Here we discuss the luminosity of electron-photon collisions that could be realized at the ILC. The photon beam can

\footnotetext{
${ }^{3}$ Signals with leptonic decays of tau leptons were studied in Ref. [29]. Because the charged leptons from tau decays tend to have a small momentum fraction, these signals also suffer from the low momentum cut of leptons.

${ }^{4}$ Another flavon signal at lepton colliders is a $t$-channel process, such as an $e^{+} e^{-} \rightarrow l^{+} l^{-}$process. In our previous paper [17], we discussed a constraint from this process at the LEP experiment [30] and showed that this constraint is weaker than the constraint from $\tau$ lepton-flavor-violating decays.
} 
TABLE II. The beam energies and luminosities to calculate flavon signals.

\begin{tabular}{lccccc}
\hline \hline & \multicolumn{3}{c}{ ILC } & upgraded ILC \\
\hline Beam energy $\left[\mathrm{GeV}^{2}\right]$ & $250 \times 200$ & $125 \times 100$ & $500 \times 400$ & $250 \times 200$ & $125 \times 100$ \\
Luminosity $\left[\mathrm{fb}^{-1}\right]$ & 300 & 300 & 4800 & 2400 & 1200 \\
\hline \hline
\end{tabular}

be obtained from an $e^{-}$beam by applying the energy spectrum of backscattered Compton photons $[18,19]$. The luminosity for $e^{-} e^{-}$can be higher than the expected $e^{+} e^{-}$ luminosity by a factor greater than 3 [31,32]. Then, the luminosity for photon-photon collisions is estimated to be $\mathcal{L}_{\gamma \gamma} \simeq \mathcal{L}_{e^{-} e^{-}} \times 3.6 \%$, where we assume a $3.6 \%$ decreasing effect from the photon energy distribution [32-34]. Since we have one photon beam for electron-photon collisions, we guess that the luminosity is roughly $\quad \mathcal{L}_{e^{-} \gamma} \sim \sqrt{0.036} \mathcal{L}_{e^{-} e^{-}} \sim 3 \sqrt{0.036} \mathcal{L}_{e^{+} e^{-}}$. Therefore, $\mathcal{L}_{e^{-\gamma} \gamma}$ would be around $50-60 \%$ of that of electron-positron collisions, and we simply apply the factor of $60 \%$ in our following analysis. For the collision energy, the energy distribution of the initial photon has a peak at around $80 \%$ of the electron energy. In our analysis we simply assume the photon energy is $80 \%$ of the electron's one. Thus, we consider electron-photon collisions with beam energies of $125 \times 100,250 \times 200$, and $500 \times 400$ where the numbers before/after $\times$ indicate the electron/photon energy in units of $[\mathrm{GeV}]$.

In this paper we calculate flavon signals with the beam energies and luminosities that are summarized in Table II. We estimate these numbers based on the planned beam energy and luminosity at the ILC [35].

\section{A. Flavor-conserving processes}

As the flavon Yukawa couplings that are proportional to $m_{\tau}$ induce large cross sections, we would expect to observe them in the process $e^{-} \gamma \rightarrow \tau^{+} \tau^{-} e^{-}$. This process mainly comes from $\varphi_{T 3}$ flavon production $e^{-} \gamma \rightarrow \tau^{-} \varphi_{T 3}$, followed by $\varphi_{T 3}$ decays into $e^{-}$and $\tau^{+}$. SM background processes are $e^{-} \gamma \rightarrow e^{-} Z / \gamma^{*}$, followed by $Z / \gamma^{*} \rightarrow \tau^{+} \tau^{-}$. Therefore, to reduce the background events, cuts on the invariant mass of a tau pair are useful. Moreover, the invariant mass of $e^{-}$ and $\tau^{+}$shows significant evidence for this signal.

In Table III we summarize the cross section of the $e^{-} \gamma \rightarrow$ $\tau^{+} \tau^{-} e^{-}$process for each beam energy. In this calculation we use MADGRAPH5 [26] and $v_{T}=2 m_{\varphi_{T 1}}=m_{\varphi_{T 2}}=$

TABLE III. The cross section of the $e^{-} \gamma \rightarrow \tau^{+} \tau^{-} e^{-}$process for each beam energy where $v_{T}=2 m_{\varphi_{T 1}}=m_{\varphi_{T 2}}=m_{\varphi_{T 3}}=$ $65 \mathrm{GeV}$. In this calculation we use a minimal $p_{T}=0.1 \mathrm{GeV}$ and a maximal $|\eta|=4.0$ for the final-state charged leptons.

\begin{tabular}{lccc}
\hline \hline Beam energy $\left[\mathrm{GeV}^{2}\right]$ & $500 \times 400$ & $250 \times 200$ & $125 \times 100$ \\
Signal cross section $[\mathrm{fb}]$ & 2.2 & 8.6 & $3.1 \times 10$ \\
Background cross & $3.0 \times 10$ & $1.1 \times 10^{2}$ & $3.6 \times 10^{2}$ \\
$\quad$ section $[\mathrm{fb}]$ & & & \\
\hline \hline
\end{tabular}

$m_{\varphi_{T 3}}=65 \mathrm{GeV}$. In this calculation we use a minimal $p_{T}=0.1 \mathrm{GeV}$ and a maximal $|\eta|=4.0$ for the final-state charged leptons. In Table III, this cross section becomes small as the beam energy becomes large, and thus we would be unlikely to detect this signal with the beam energy $500 \times 400 \mathrm{GeV}^{2}$.

Hereinafter, we study event selection cuts to obtain sufficient discovery significance. We perform detector simulations using MadGraph5 [26], Pythia [27], and Delphes [28]. First, we generate events for the signal process and SM background process, and perform the detector simulations based on the ILC detector proposal [36]. ${ }^{5}$ Next, we count the number of events which satisfy our selection rules. Our first selection rule is whether an event contains two tau jets $\tau_{h}$ and one electron $e^{-}$. The second selection rule involves applying a cut on the invariant mass of the tau-jet pair $m_{\tau_{h}^{-} \tau_{h}^{+}}$to reduce the events which come from the SM. Finally, to collect the events which come from the signal process, we use the invariant masses of $e^{-}$and $\tau_{h}^{+} m_{e^{-}} \tau_{h}^{+}$.

The left panel of Fig. 2 shows the $m_{\tau_{h}^{-} \tau_{h}^{+}}$distribution after the first selection where the beam energy is $250 \times 200 \mathrm{GeV}^{2}$. In this calculation we consider only the signal process and generate 10 times the expected number of events at the ILC. This distribution has a jump at $70 \mathrm{GeV}$. This jump comes from the $p_{T}$ cut $p_{T}\left(\tau_{h}^{+}\right) \leq 10 \mathrm{GeV}$. The right panel of Fig. 2 shows the distribution where the horizontal axis is $p_{T}\left(\tau^{+}\right)$and the vertical axis is $m_{\tau^{-} \tau^{+}}$in a parton-level simulation. As with the left plot, we consider only the signal process and generate 10 times the expected number of events at the ILC. This picture shows that $p_{T}\left(\tau^{+}\right)$and $m_{\tau^{-}} \tau^{+}$are positively correlated because, if $\tau^{+}$travels in the same direction as $\tau^{-}$, $m_{\tau^{-} \tau^{+}}$is small and $p_{T}\left(\tau^{+}\right)$is small to realize the momentum conservation. Therefore, for $p_{T}\left(\tau^{+}\right)>10 \mathrm{GeV}$ there are a few events which have small $m_{\tau^{-} \tau^{+}} .^{6}$ On the other hand, in

\footnotetext{
${ }^{5}$ In this paper, we use a default tau-tagging efficiency of $40 \%$ (as in Ref. [36]). In reality, at the ILC the tau-tagging efficiency may be higher; for example, in Ref. [37] the tau-tagging efficiency was quoted as $60 \%$. The expected event numbers for the events with $n \tau$ 's would be multiplied by roughly the $n$th power of the enhancement rate of the tau-tagging efficiency.

${ }^{6}$ Because this picture comes from the parton-level simulation, $p_{T}\left(\tau^{+}\right)$is the tau-lepton transverse momentum. On the other hand, in the detector-level simulation transverse momentum cuts are applied to the tau hadron. Although there is a difference between the transverse momenta of the tau lepton and tau hadron, we can understand the effect of $p_{T}\left(\tau_{h}^{+}\right)$cuts on $m_{\tau_{h}^{-}} \tau_{h}^{+}$.
} 

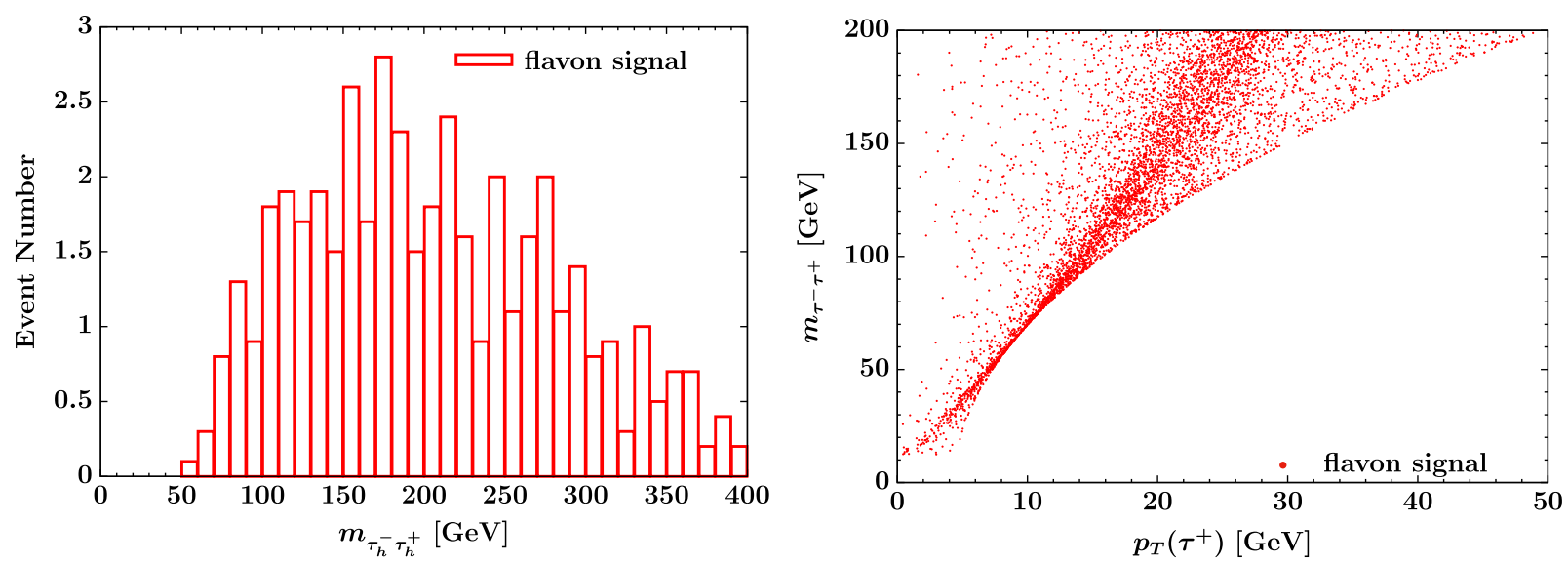

FIG. 2. The $m_{\tau_{h}^{-} \tau_{h}^{+}}$distribution after the first selection (left) and the distribution, where the horizontal axis is the transverse momentum of an antitau lepton $p_{T}\left(\tau^{+}\right)$and the vertical axis is the $m_{\tau^{-} \tau^{+}}$in a parton-level simulation (right). In these calculations we consider only the signal process and generate 10 times the expected number of events at the ILC.

the SM background process the $m_{\tau_{h}^{-} \tau_{h}^{+}}$distribution has a peak at around the $Z$-boson mass $M_{Z}$. For these reasons, our second selection rule works well to select signal events in our model.

In Table IV we summarize the ratios of the signal event rate and background event rate. Here the event rate means the ratio of the number of events after the cuts to the number of events before the cuts. Moreover, we summarize the ratios of the number of signal events and background events, and the significances of the signal after applying our selection cuts at the ILC and upgraded ILC. As we calculate in Table III, the flavon masses are fixed to $v_{T}=2 m_{\varphi_{T 1}}=$ $m_{\varphi_{T 2}}=m_{\varphi_{T 3}}=65 \mathrm{GeV}$ in this calculation. The background event number is strongly suppressed, and therefore we use a significance which is based on the Poisson distribution $S_{c L}$ [38]. $S_{c L}$ is defined by

$$
S_{c l}=\sqrt{2\left(\left(N_{S}+N_{B G}\right) \ln \left(1+N_{S} / N_{B G}\right)-N_{S}\right)},
$$

where $N_{S}$ is the signal event number, and $N_{B G}$ is the background event number. In our second selection rule, we remove events with larger $m_{\tau_{h}^{-} \tau_{h}^{+}}$as the beam energy becomes larger. This is because the $m_{\tau_{h}^{-}} \tau_{h}^{+}$jump position which we showed in Fig. 2 becomes higher as the beam energy becomes larger. Table IV shows that when the beam energy is $250 \times 200 \mathrm{GeV}^{2}$ and $125 \times 100 \mathrm{GeV}^{2}$ we can expect sufficient discovery significance for the flavon mass around the lower limit. When the beam energy is $500 \times 400 \mathrm{GeV}^{2}$, the discovery significance is sufficient even though the signal cross section is small, because at the upgraded ILC we can expect large integrated luminosity.

TABLE IV. Ratios of the signal event rate and background event rate $\left(R_{S} / R_{B G}\right)$, ratios of the signal event number and background event number $\left(N_{S} / N_{B G}\right)$, and significances of the signal $S_{c L}$ after applying our selection rules at the ILC and upgraded ILC. In this calculation we use $v_{T}=2 m_{\varphi_{T 1}}=m_{\varphi_{T 2}}=m_{\varphi_{T 3}}=65 \mathrm{GeV}$.

\begin{tabular}{lccr}
\hline \hline $250 \times 200\left[\mathrm{GeV}^{2}\right]$ & $R_{S}[\%] R_{B G}[\%]$ & $N_{S} / N_{B G}$ & $S_{c L}$ \\
\hline contain $\tau_{h}^{+} \tau_{h}^{-} e^{-}$ & $1.7 / 1.8$ & $45 / 590$ & 1.8 \\
$m_{\tau_{h}^{-} \tau_{h}^{+}}>M_{Z}+15.0[\mathrm{GeV}]$ & $1.6 / 0.15$ & $41 / 49$ & 5.3 \\
$30[\mathrm{GeV}]<m_{e^{-} \tau_{h}^{+}}<65[\mathrm{GeV}]$ & $1.5 / 0.00093$ & $38 / 3.1$ & 12 \\
\hline \hline $125 \times 100\left[\mathrm{GeV}^{2}\right]$ & $R_{S}[\%] R_{B G}[\%]$ & $N_{S} / N_{B G}$ & $S_{c L}$ \\
\hline contain $\tau_{h}^{+} \tau_{h}^{-} e^{-}$ & $1.8 / 1.9$ & $170 / 2100$ & 3.7 \\
$m_{\tau_{h}^{-} \tau_{h}^{+}}>M_{Z}+5.0[\mathrm{GeV}]$ & $1.0 / 0.089$ & $96 / 96$ & 8.6 \\
$25[\mathrm{GeV}]<m_{e^{-} \tau_{h}^{+}}<70[\mathrm{GeV}]$ & $1.0 / 0.028$ & $95 / 31$ & 13 \\
& & & $N_{S} / N_{B G}$ \\
\hline \hline $500 \times 400\left[\mathrm{GeV}^{2}\right]$ & $R_{S}[\%] R_{B G}[\%]$ & $97 / 2100$ & 2.1 \\
\hline $\operatorname{contain} \tau_{h}^{+} \tau_{h}^{-} e^{-}$ & $0.91 / 1.4$ & $72 / 170$ & 5.2 \\
$m_{\tau_{h}^{-} \tau_{h}^{+}}>290[\mathrm{GeV}]$ & $0.68 / 0.12$ & $72 / 5.2$ & 17 \\
$25[\mathrm{GeV}]<m_{e^{-} \tau_{h}^{+}}<70[\mathrm{GeV}]$ & $0.67 / 0.0036$ &
\end{tabular}



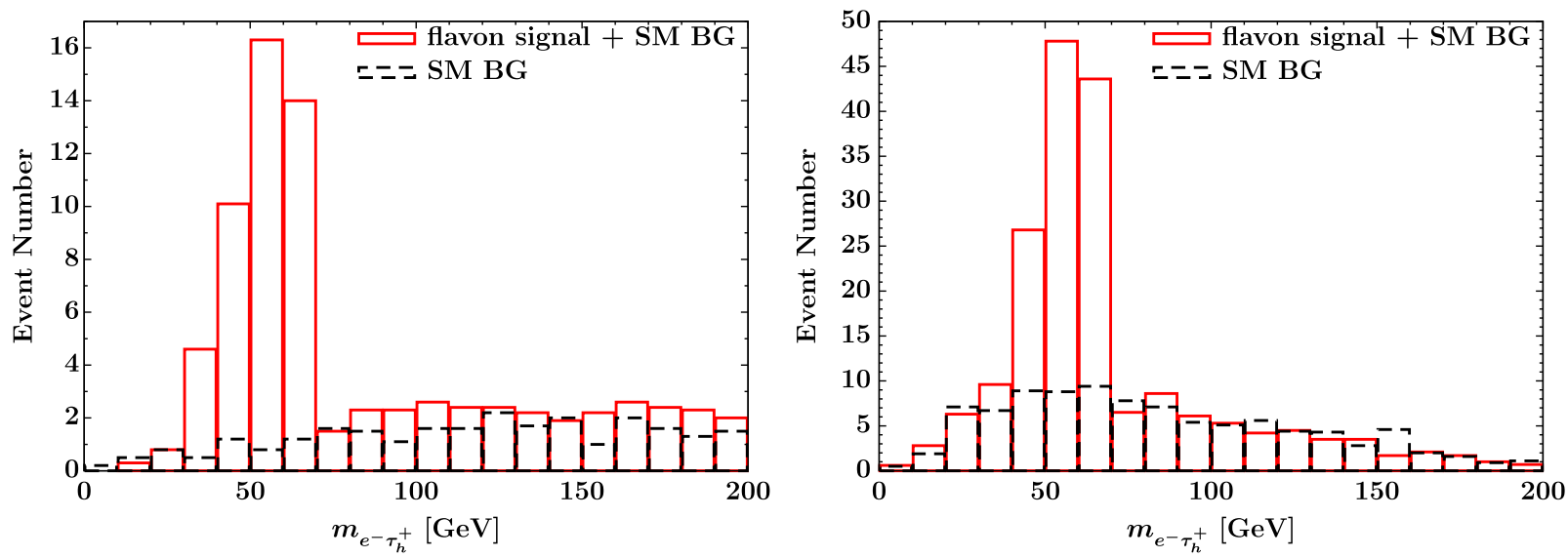

FIG. 3. The $m_{e^{-}} \tau^{+}$distribution after the second selection rule for the beam energies $250 \times 200 \mathrm{GeV}^{2}$ (left) and $125 \times 100 \mathrm{GeV}^{2}$ (right) at the ILC. The red solid histograms are for both the SM and flavon processes, while the black dashed histograms are for only the SM process. In this calculation we fix the flavon masses to be $v_{T}=2 m_{\varphi_{T 1}}=m_{\varphi_{T 2}}=m_{\varphi_{T 3}}=65 \mathrm{GeV}$.

The left and right panels in Fig. 3 show the $m_{e^{-}} \tau_{h}^{+}$ distribution after the second selection rule for the beam energies $250 \times 200 \mathrm{GeV}^{2}$ and $125 \times 100 \mathrm{GeV}^{2}$ at the ILC, respectively. The red solid histograms are for both the SM and flavon processes, while the black dashed histograms are for only the SM process. In this calculation we fix the flavon masses to be $v_{T}=2 m_{\varphi_{T 1}}=m_{\varphi_{T 2}}=m_{\varphi_{T 3}}=65 \mathrm{GeV}$. Both figures show a clear peak around the flavon mass. Figure 4 is the same as Fig. 3, but for the beam energy $500 \times 400 \mathrm{GeV}^{2}$ at the upgraded ILC. This figure also show a clear peak around the flavon mass. From Table IV, the significance for the beam energy $500 \times 400 \mathrm{GeV}^{2}$ is not larger than those for the beam energies $250 \times$ $200 \mathrm{GeV}^{2}$ and $125 \times 100 \mathrm{GeV}^{2}$ after the second selection rule. On the other hand, the peak around the flavon mass for

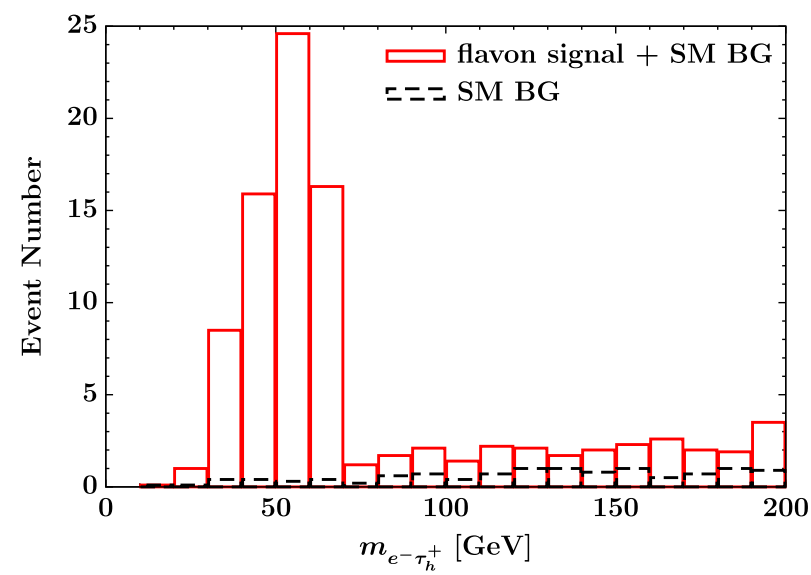

FIG. 4. The $m_{e^{-}} \tau_{h}^{+}$distribution where the beam energy is $500 \times$ $400 \mathrm{GeV}^{2}$ at the upgraded ILC. The red solid histograms indicate the number of events which come from the SM and flavon interactions, and the black dashed histograms indicate the number of events which come from only the SM interactions. In this calculation the flavon masses are $v_{T}=2 m_{\varphi_{T 1}}=m_{\varphi_{T 2}}=m_{\varphi_{T 3}}=$ $65 \mathrm{GeV}$. the beam energy $500 \times 400 \mathrm{GeV}^{2}$ is clearer than those for the beam energies $250 \times 200 \mathrm{GeV}^{2}$ and $125 \times 100 \mathrm{GeV}^{2}$. This is because the number of events in the small$m_{e^{-} \tau^{+}}$region is tiny in the SM for the beam energy $500 \times 400 \mathrm{GeV}^{2}$. This feature comes from the antitauhadron transverse momentum cuts as we showed above.

Finally, we study the discovery significance for different flavon masses at the upgraded ILC. We consider the flavon masses $v_{T}=2 m_{\varphi_{T 1}}=m_{\varphi_{T 2}}=m_{\varphi_{T 3}}=100,150$, and $200 \mathrm{GeV}$. When the beam energy is $250 \times 200 \mathrm{GeV}^{2}$, we find sufficient discovery significance for the flavon masses 100 and $150 \mathrm{GeV}$. When the beam energy is $125 \times 100 \mathrm{GeV}^{2}$, we find sufficient discovery significance for the flavon mass $100 \mathrm{GeV}$. Figure 5 shows the $m_{e^{-}} \tau_{h}^{+}$distribution at the upgraded ILC. The left panel is for the flavon mass $150 \mathrm{GeV}$ and the beam energy $250 \times 200 \mathrm{GeV}^{2}$, and the right panel is for the flavon mass $100 \mathrm{GeV}$ and the beam energy $125 \times 100 \mathrm{GeV}^{2}$. We expect sufficient discovery significance for flavon masses lighter than $150 \mathrm{GeV}$.

\section{B. Flavor-violating processes}

In our model, there are the following three lepton-flavorviolating processes: $e^{-} \gamma \rightarrow \tau^{+} \mu^{-} \mu^{-}, \mu^{+} \tau^{-} \tau^{-}$, and $e^{+} \tau^{-} \mu^{-}$. The amplitudes of these flavor-violating processes are proportional to at least two charged lepton masses. The cross section for the $e^{+} \tau^{-} \mu^{-}$final state is smaller than the cross sections for the other two final states, because this process comes from the coupling which is proportional to $m_{e}$. Therefore in this paper we consider $e^{-} \gamma \rightarrow \tau^{+} \mu^{-} \mu^{-}$and $e^{-} \gamma \rightarrow \mu^{+} \tau^{-} \tau^{-}$processes. The cross sections for both final states are nearly the same. Because these processes induce lepton flavor violation, SM contributions are extremely suppressed. Therefore even if we detect a few events for these final states, it can be a strong evidence for our model.

In Table V we summarize the cross sections of the $e^{-} \gamma \rightarrow$ $\tau^{+} \mu^{-} \mu^{-}$process for each beam energy. Here we adopt 

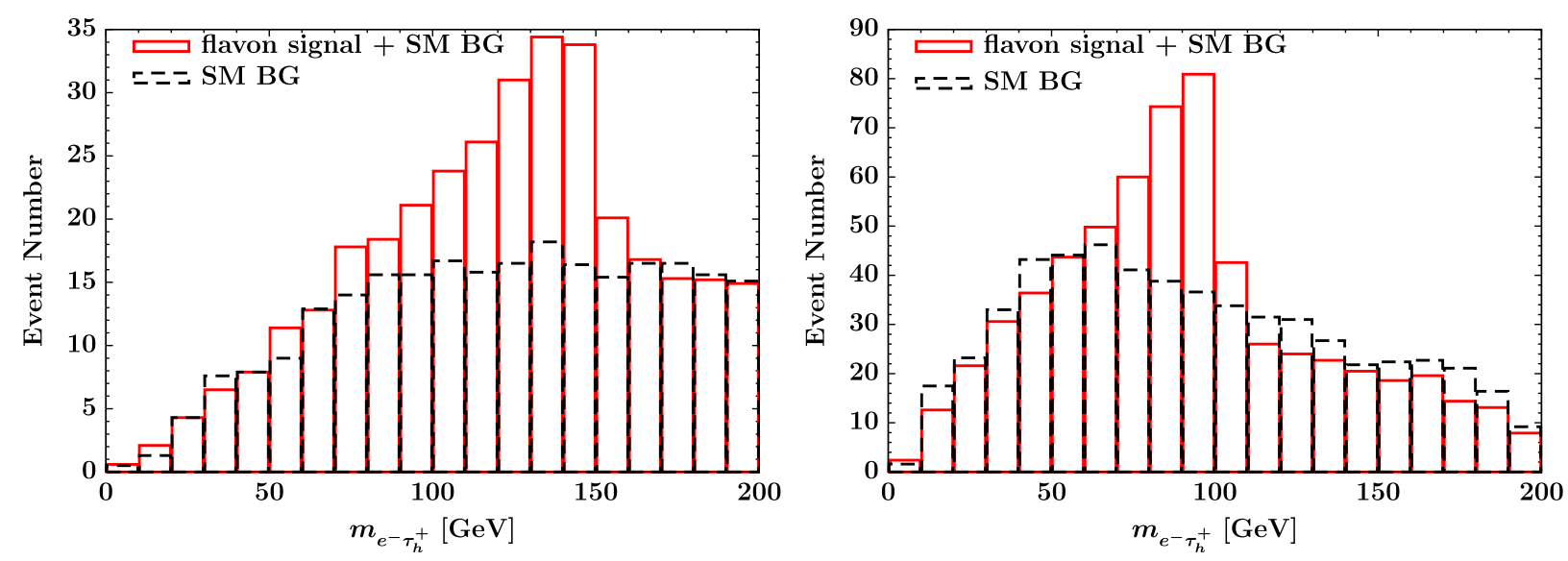

FIG. 5. The $m_{e^{-} \tau_{h}^{+}}$distribution where the beam energies are $250 \times 200 \mathrm{GeV}^{2}$ (left) and $125 \times 100 \mathrm{GeV}^{2}$ (right) at the upgraded ILC. The red solid histograms indicate the number of events which come from the SM and flavon interactions, and the black dashed histograms indicate the number of events which come from only the SM interactions. In this calculation the flavon masses are $v_{T}=2 m_{\varphi_{T 1}}=m_{\varphi_{T 2}}=m_{\varphi_{T 3}}=150 \mathrm{GeV}$ (left) and $100 \mathrm{GeV}$ (right).

$v_{T}=2 m_{\varphi_{T 1}}=m_{\varphi_{T 2}}=m_{\varphi_{T 3}}=65 \mathrm{GeV}$. The cross sections are calculated using MADGRAPH5 [26] with the following kinematical cuts: a minimal $p_{T}$ is $0.1 \mathrm{GeV}$, and a maximal $|\eta|$ is 4.0 for the final-state charged leptons. Table $\mathrm{V}$ shows that around the flavon lower mass limit the $250 \times 200$ and $125 \times 100 \mathrm{GeV}^{2}$ beam energies are favored for detecting the flavor-violating processes. Moreover, when $O(10-100) \mathrm{fb}^{-1}$ data are collected, we will detect these lepton-flavor-violating processes at the ILC.

As we mentioned in Sec. III, the identification of tau leptons is not straightforward. Our signal processes have a tau lepton, and therefore we perform detector simulations. We generate $10^{4}$ events for each process using MADGRAPH5 [26] and select events with three charged leptons and lepton flavor violation. For the detector simulations we use Pythia [27] and DelPHES [28] based on the ILC detector proposal [36]. In Table VI we summarize event rates and expected event numbers for each process at the ILC and upgraded ILC. In this table we consider the $250 \times 200$ and $125 \times 100 \mathrm{GeV}^{2}$ beam energies and $v_{T}=2 m_{\varphi_{T 1}}=m_{\varphi_{T 2}}=m_{\varphi_{T 3}}=65 \mathrm{GeV}$. In the $\mu^{+} \tau^{-} \tau^{-}$mode there are final states which include $e^{-}$, such as $\mu^{+} \tau^{-} e^{-}$and $\mu^{+} e^{-} e^{-}$. However these final states have large SM background which comes from flavor-conserving processes $e^{-} \gamma \rightarrow \tau^{+} \tau^{-} e^{-}$. Therefore in this calculation we ignore these final states. This table shows that around the flavon mass lower limit observations of the lepton-flavor-violating signals are expected at the ILC. Moreover, at the upgraded ILC we can expect to observe about 20 (40) flavor-violating events for the

TABLE V. The cross sections of the $e^{-} \gamma \rightarrow \tau^{+} \mu^{-} \mu^{-}$process for each beam energy where $v_{T}=2 m_{\varphi_{T 1}}=m_{\varphi_{T 2}}=$ $m_{\varphi_{T 3}}=65 \mathrm{GeV}$. In this calculation we use a minimal $p_{T}=0.1 \mathrm{GeV}$, and a maximal $|\eta|=4.0$ for the final-state charged leptons.

\begin{tabular}{|c|c|c|c|}
\hline Beam energy $\left[\mathrm{GeV}^{2}\right]$ & $500 \times 400$ & $250 \times 200$ & $125 \times 100$ \\
\hline Cross section $[\mathrm{fb}]$ & $7.9 \times 10^{-3}$ & $3.1 \times 10^{-2}$ & $1.1 \times 10^{-1}$ \\
\hline
\end{tabular}

TABLE VI. Event rates and expected event numbers for each final state where the beam energy is $250 \times 200 \mathrm{GeV}^{2}\left(125 \times 100 \mathrm{GeV}^{2}\right)$ and $v_{T}=2 m_{\varphi_{T 1}}=m_{\varphi_{T 2}}=m_{\varphi_{T 3}}=65 \mathrm{GeV}$ at the ILC and upgraded ILC.

\begin{tabular}{|c|c|c|c|c|c|}
\hline & Cross section $[\mathrm{fb}]$ & $\begin{array}{l}\text { Final } \\
\text { state }\end{array}$ & $\begin{array}{c}\text { Event rate } \\
{[\%]}\end{array}$ & $\begin{array}{l}\text { Event number at } \\
300(300)\left[\mathrm{fb}^{-1}\right]\end{array}$ & $\begin{array}{l}\text { Event number at } \\
2400(1200)\left[\mathrm{fb}^{-1}\right]\end{array}$ \\
\hline \multirow[t]{4}{*}{$\tau^{+} \mu^{-} \mu^{-}$mode } & $3.1 \times 10^{-2}\left(1.1 \times 10^{-1}\right)$ & sum & $19(23)$ & $1.8(7.4)$ & $14(30)$ \\
\hline & & $\tau_{h}^{+} \mu^{-} \mu^{-}$ & $6.5(8.9)$ & $0.59(2.9)$ & $4.8(12)$ \\
\hline & & $\mu^{+} \mu^{-} \mu^{-}$ & $6.5(7.2)$ & $0.59(2.3)$ & $4.7(9.3)$ \\
\hline & & $e^{+} \mu^{-} \mu^{-}$ & $6.5(6.8)$ & $0.60(2.2)$ & $4.8(8.8)$ \\
\hline \multirow[t]{4}{*}{$\mu^{+} \tau^{-} \tau^{-}$mode } & $3.1 \times 10^{-2}\left(1.1 \times 10^{-1}\right)$ & sum & $6.5(7.1)$ & $0.59(2.3)$ & $4.8(9.2)$ \\
\hline & & $\mu^{+} \tau_{h}^{-} \mu^{-}$ & $3.2(3.5)$ & $0.29(1.1)$ & $2.3(4.6)$ \\
\hline & & $\mu^{+} \tau_{h}^{-} \tau_{h}^{-}$ & $2.2(2.3)$ & $0.20(0.76)$ & $1.6(3.0)$ \\
\hline & & $\mu^{+} \mu^{-} \mu^{-}$ & $1.1(1.2)$ & $0.10(0.40)$ & $0.84(1.6)$ \\
\hline
\end{tabular}




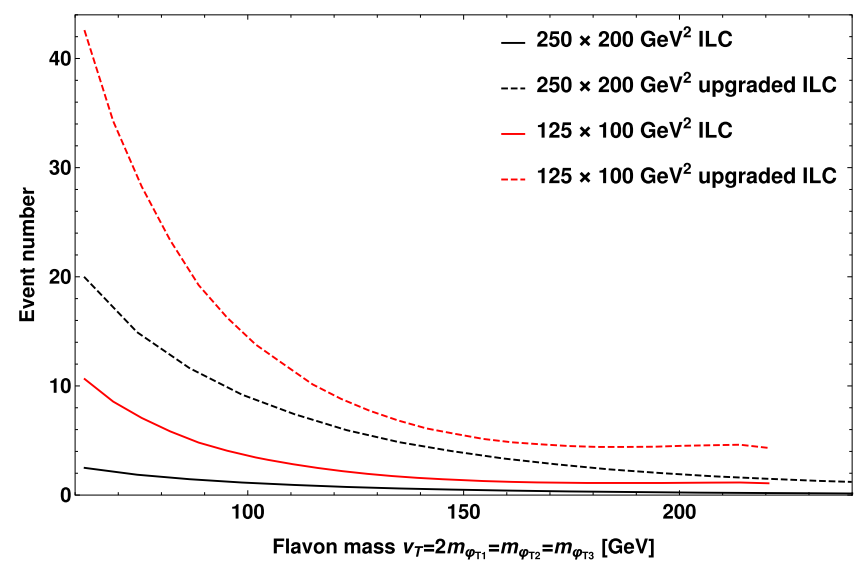

FIG. 6. The flavon mass dependence for the event number of the lepton-flavor-violating process where the beam energy and luminosity is $250 \times 200 \mathrm{GeV}^{2}$ at the ILC (black solid line), $125 \times 100 \mathrm{GeV}^{2}$ at the ILC (red solid line), $250 \times 200 \mathrm{GeV}^{2}$ at the upgraded ILC (black dashed line), and $125 \times 100 \mathrm{GeV}^{2}$ at the upgraded ILC (red dashed line).

$250 \times 200 \mathrm{GeV}^{2}\left(125 \times 100 \mathrm{GeV}^{2}\right)$ beam energy. If many flavor-violating events are observed, we can test this model by using a ratio of the event numbers for each process.

In this subsection we do not consider background events from the SM interactions. Because these signals have large lepton flavor violation, for example, the $e^{-} \gamma \rightarrow \tau^{+} \mu^{-} \mu^{-}$ process induces $\Delta N_{e}=-1, \Delta N_{\mu}=+2$, and $\Delta N_{\tau}=-1$. In the SM, lepton flavor violations are induced via the weak interactions, and the weak interactions produce neutrinos. Therefore even if the number of background events from the SM interactions is sizable, we can identify signals by using a missing momentum cut.

Figure 6 shows the flavon mass dependence for the sum of the event numbers of the flavor-violating signals. In this calculation we assume that the beam energies are $250 \times 200$ and $125 \times 100 \mathrm{GeV}^{2}$. The black solid, red solid, black dashed, and red dashed lines show the event numbers for the $250 \times 200 \mathrm{GeV}^{2}$ beam energy at the ILC, $125 \times 100 \mathrm{GeV}^{2}$ beam energy at the ILC, $250 \times 200 \mathrm{GeV}^{2}$ beam energy at the upgraded ILC, and $125 \times 200 \mathrm{GeV}^{2}$ beam energy at the upgraded ILC, respectively. The red lines have a sharp cutoff at around $m_{\varphi_{T 2}}=m_{\varphi_{T 3}}=225 \mathrm{GeV}$, because of the loss of the phase space. We can expect a greater number of events for the $125 \times 100 \mathrm{GeV}^{2}$ beam energy than for the $250 \times 200 \mathrm{GeV}^{2}$ beam energy, while we can search for wider mass regions with the $250 \times 200 \mathrm{GeV}^{2}$ beam energy.

If we assume that the number of background events is one, the minimal number of signal events which gives $S_{c L}=3$ is four. We estimate the mass upper limits that satisfy $S_{c L}=3$ for any flavon mass. At the ILC, it is not possible to satisfy $S_{C L}=3$ when the beam energy is $250 \times 200 \mathrm{GeV}^{2}$. On the other hand, when the beam energy is $125 \times 100 \mathrm{GeV}^{2}$ it is possible to satisfy $S_{c L}=3$ below the flavon mass $m_{\varphi_{T 2}}=m_{\varphi_{T 3}}=96 \mathrm{GeV}$. In the upgraded ILC case when the beam energy is $250 \times 200 \mathrm{GeV}^{2}$, it is possible to satisfy $S_{c L}=3$ below the flavon mass $m_{\varphi_{T 2}}=m_{\varphi_{T 3}}=150 \mathrm{GeV}$. Moreover, when the beam energy is $125 \times 100 \mathrm{GeV}^{2}$ it is possible to satisfy $S_{c L}=3$ below the cross section decreases at a flavon mass of around $m_{\varphi_{T 2}}=m_{\varphi_{T 3}}=225 \mathrm{GeV}$.

\section{SUMMARY AND DISCUSSION}

In this paper, we discussed collider signals of the light flavons $\varphi_{T}$ which were introduced in the modified $\mathrm{AF}$ model. At the LHC and in electron-positron collisions at the ILC, we cannot obtain sufficient discovery significance. We found that electron-photon collisions at the ILC have advantages for searching for signals where flavons are produced, as $e^{-} \gamma \rightarrow l^{-} \varphi_{T}$ and decay into two charged leptons. Then, we analyzed signals of the flavor-conserving final state $\tau^{+} \tau^{-} e^{-}$and flavor-violating final states $\tau^{+} \mu^{-} \mu^{-}$ and $\mu^{+} \tau^{-} \tau^{-}$by carrying out numerical simulations. For the former final state, SM background can be strongly suppressed by imposing cuts on the invariant masses of the final-state leptons. As a result, around the flavon mass lower limit we can obtain sufficient discovery significance for each beam energy. Moreover, at the upgraded ILC we expect sufficient discovery significance for flavon masses lighter than $150 \mathrm{GeV}$. For the latter final states, we found that at the ILC we can expect signal observation, and at the upgraded ILC we can expect sufficient signal observation to test this model. In particular, at the upgraded ILC we expect sufficient discovery significance for flavon masses lighter than $225 \mathrm{GeV}$. Therefore, stronger flavon mass constraints could be obtained from future collider experiments compared to those from the flavor-violating decay of leptons.

\section{ACKNOWLEDGMENTS}

Y.M. is supported in part by the National Natural Science Foundation of China (NNSFC) under Contracts No. 11435003, No. 11225523 and No. 11521064. Y. M. and Y.S. are supported in the part by the National Research Foundation of Korea (NRF) Research Grant No. NRF-2015R1A2A1A05001869. Y. S. is supported in part by JSPS Grant-in-Aid for Scientific Research No. 16J05332. 
[1] F. P. An et al. (DAYA-BAY Collaboration), Phys. Rev. Lett. 108, 171803 (2012).

[2] J. K. Ahn et al. (RENO Collaboration), Phys. Rev. Lett. 108, 191802 (2012).

[3] P. Adamson et al. (MINOS Collaboration), Phys. Rev. Lett. 110, 251801 (2013).

[4] Y. Abe et al. (Double Chooz Collaboration), Phys. Lett. B 735, 51 (2014).

[5] K. Abe et al. (T2K Collaboration), Phys. Rev. Lett. 112, 061802 (2014).

[6] H. Ishimori, T. Kobayashi, H. Ohki, Y. Shimizu, H. Okada, and M. Tanimoto, Prog. Theor. Phys. Suppl. 183, 1 (2010).

[7] H. Ishimori, T. Kobayashi, H. Ohki, H. Okada, Y. Shimizu, and M. Tanimoto, Lect. Notes Phys. 858, 87 (2012).

[8] H. Ishimori, T. Kobayashi, Y. Shimizu, H. Ohki, H. Okada, and M. Tanimoto, Fortschr. Phys. 61, 441 (2013).

[9] S. F. King, A. Merle, S. Morisi, Y. Shimizu, and M. Tanimoto, New J. Phys. 16, 045018 (2014).

[10] G. Altarelli and F. Feruglio, Nucl. Phys. B720, 64 (2005).

[11] G. Altarelli and F. Feruglio, Nucl. Phys. B741, 215 (2006).

[12] Y. Shimizu, M. Tanimoto, and A. Watanabe, Prog. Theor. Phys. 126, 81 (2011).

[13] M. Holthausen, M. Lindner, and M. A. Schmidt, Phys. Rev. D 87, 033006 (2013).

[14] I. de Medeiros Varzielas, O. Fischer, and V. Maurer, J. High Energy Phys. 08 (2015) 080.

[15] T. Kobayashi, Y. Omura, F. Takayama, and D. Yasuhara, J. High Energy Phys. 10 (2015) 042.

[16] I. d. M. Varzielas and O. Fischer, J. High Energy Phys. 01 (2016) 160.

[17] Y. Muramatsu, T. Nomura, and Y. Shimizu, J. High Energy Phys. 03 (2016) 192.

[18] I. F. Ginzburg, G. L. Kotkin, S. L. Panfil, V. G. Serbo, and V. I. Telnov, Nucl. Instrum. Methods Phys. Res., Sect. A 219, 5 (1984).

[19] I. F. Ginzburg and G. L. Kotkin, Eur. Phys. J. C 13, 295 (2000).
[20] I. F. Ginzburg, G. L. Kotkin, V. G. Serbo, and V. I. Telnov, Pis'ma Zh. Eksp. Teor. Fiz. 34, 514 (1981) [JETP Lett. 34, 491 (1981)].

[21] I. F. Ginzburg, G. L. Kotkin, V. G. Serbo, and V. I. Telnov, Nucl. Instrum. Methods Phys. Res. 205, 47 (1983).

[22] S. Kanemura and K. Tsumura, Phys. Lett. B 674, 295 (2009).

[23] C. X. Yue, H. D. Yang, and H. L. Feng, J. Phys. G 37, 015006 (2010).

[24] T. Morozumi, H. Okane, H. Sakamoto, Y. Shimizu, K. Takagi, and H. Umeeda, arXiv:1707.04028.

[25] S. Pascoli and Y. L. Zhou, J. High Energy Phys. 10 (2016) 145.

[26] J. Alwall, R. Frederix, S. Frixione, V. Hirschi, F. Maltoni, O. Mattelaer, H.-S. Shao, T. Stelzer, P. Torrielli, and M. Zaro, J. High Energy Phys. 07 (2014) 079.

[27] T. Sjostrand, S. Mrenna, and P. Z. Skands, J. High Energy Phys. 05 (2006) 026.

[28] J. de Favereau, C. Delaere, P. Demin, A. Giammanco, V. Lemaître, A. Mertens, and M. Selvaggi (DELPHES 3 Collaboration), J. High Energy Phys. 02 (2014) 057.

[29] S. Kanemura, K. Tsumura, and H. Yokoya, Phys. Rev. D 85, 095001 (2012).

[30] LEP, ALEPH, DELPHI, L3, and OPAL Collaborations, and LEP Electroweak Working Group ,arXiv:hep-ex/0312023.

[31] V. I. Telnov, Nucl. Instrum. Methods Phys. Res., Sect. A 355, 3 (1995).

[32] K. Fujii et al. (LCC Physics Working Group Collaboration), arXiv:1607.03829.

[33] B. Badelek et al. (ECFA/DESY Photon Collider Working Group Collaboration), Int. J. Mod. Phys. A 19, 5097 (2004).

[34] F. Richard, arXiv:1604.01640.

[35] T. Barklow, J. Brau, K. Fujii, J. Gao, J. List, N. Walker, and K. Yokoya, arXiv:1506.07830.

[36] DeLphes silicon detector for the International Linear Collider (ILC), a detector card for the fast detector simulator DELPHES, https://dsid.hepforge.org/.

[37] H. Baer et al., arXiv:1306.6352.

[38] G. L. Bayatian et al. (CMS Collaboration), J. Phys. G 34, 995 (2007). 